Result We identified 4069 cases of UC over the study period. There were 1678 never smokers (41\%), 329 smokers (8\%), 1541 ex-smokers (38\%) and 521 patients whose follow-up smoking status changed (13\%). Multivariate regression analysis, adjusting for all covariates listed in table 1, demonstrated smokers had a significantly lower risk of thiopurine use compared to both never smokers (HR 0.52, 95\% CI 0.27-0.97, $\mathrm{p}=0.04$ ) and ex-smokers (HR 0.51, 95\% CI 0.27-0.98, $\mathrm{p}=0.04)$. In contrast there was no difference in corticosteroid dependency (OR 0.83 , 95\% CI $0.44-1.75$ ) or rates of colectomy (HR 0.4 , 95\% CI 0.53-3.02) in a multivariate analysis. Conclusions Smoking is associated with a decreased requirement for thiopurines however it does not impact on the risk of corticosteroid dependency or colectomy. The risks associated with smoking outweigh any benefits and smoking cessation should be encouraged.

\section{PWE-007 A SYSTEMATIC REVIEW OF OUTCOMES AND ADVERSE EVENTS FOR RANDOMISED CONTROLLED TRIALS IN CROHN'S DISEASE}

${ }^{1}$ Ms Heather Catt*, ${ }^{2}$ Dyfrig Hughes, ${ }^{1,3}$ Keith Bodger, ${ }^{1}$ Jamie Kirkham. ${ }^{1}$ University Of Liverpool (MRC Trials Hub), Liverpool, UK; ${ }^{2}$ University of Bangor, Bangor, UK; ${ }^{3}$ Aintree University Hospital NHS Trust, Liverpool, UK

\subsection{6/gutjnl-2018-BSGAbstracts.139}

Introduction Despite major progress in drug development for Crohn's disease (CD) and advances in trial methodology, there is no internationally recognised core outcome set (COS). Poor standardisation in outcome reporting may impact negatively on translation of trials into practice. The suitability of traditional disease activity indices as primary end-points has been challenged, with growing interest in objective measures of inflammation. We undertook a systematic review to explore heterogeneity and time trends in the reporting of efficacy and safety outcomes in placebo-controlled randomised controlled trials (RCTs) of patients with CD.

Methods We searched MEDLINE, EMBASE, CINAHL and Cochrane Library from their inception to November 2015, for RCTs of adult CD patients with treated with medical or surgical therapies. We extracted information on efficacy and safety outcomes, definitions of end-points, and measurement instruments. To explore temporal trends studies were stratified by publication date (pre-2009 and 2009-onwards).

Results 181 RCTs comprising 23850 patients. Trial focus: Induction of remission, 110 trials (60.8\%), 104 medical and 6 surgical interventions. Maintenance of remission, 71 trials (39.2\%). Biologics were intervention of interest in $33.7 \%$, as either monotherapy or part of a combination therapy. 92.3\% of trials reported clinical efficacy outcomes as a primary or secondary endpoint. CDAI was the dominant index, used to determine clinical response or remission in $63.5 \%$ of trials. However, there was heterogeneity, with 35 definitions of response or remission. CDAI $<150$ was the commonest endpoint, but reporting reduced between periods $(46.4 \%$ to $41.1 \%$ of trials), whilst CDAI100 reporting increased $(16.8 \%$ to $30.4 \%)$. Reporting of objective measures of inflammation increased over time, but with lack of standardisation. Reporting of both histologic and endoscopic outcomes increased, from $3.2 \%$ to $12.5 \%$ and from $14.4 \%$ to $30.4 \%$ of RCTs, respectively. Biomarker reporting increased from $33.3 \%$ to
$40.6 \%$ of trials. Patient-reported outcome measures (PROMs) were reported in $41.4 \%$ of trials with growth in reporting from $39.2 \%$ to $46.4 \%$. Safety outcomes were reported explicitly in $35.4 \%$ of trials and reporting increased from $32.8 \%$ to $41.1 \%$.

Conclusions As expected, the CDAI was the dominant composite index reported but there was significant variation in the selection and definition of clinical trial end-points in RCTs for CD between studies, and over time. Despite growth in reporting of objective measures of inflammation and in PROMs, there is much heterogeneity and lack of standardisation. This highlights the need for international researchers and clinicians to develop a COS for comparative effectiveness research in CD.

\section{PWE-008 CLINICAL OUTCOMES OF USTEKINUMAB IN RESISTANT CROHN'S DISEASE: UK IBD TERTIARY REFERRAL CENTRE 'REAL-WORLD' EXPERIENCE}

Simon Peter Borg-Bartolo*, Karen Kemp, Robert P Willert, Alistair J Makin, Scott Edward Levison. Department of Gastroenterology, Manchester Royal Infirmary, Manchester University NHS Foundation Trust, Manchester, UK

\subsection{6/gutjnl-2018-BSGAbstracts. 140}

Introduction Ustekinumab (UST) binds to the p40 subunit of IL12 and IL23 to prevent IL12RB1 cell-surface receptor activation and thus inhibits downstream inflammatory signalling. It is approved for moderately to severely active Crohn's disease (NICE TA456). We assessed the clinical outcomes and safety of UST in a 'real-world' cohort of refractory Crohn's disease patients treated at a single UK centre.

Methods We retrospectively collected data from the electronic records of Crohn's disease patients treated with UST at a single UK IBD tertiary referral centre. Patient demographics and adverse events were recorded. Clinical response to UST was evaluated at baseline and follow up using Harvey-Bradshaw Index (HBI) scores, $\mathrm{C}$ reactive protein (CRP), and faecal calprotectin (FC). Paired Student's T Tests were used to determine statistical significance.

Results 26 patients (mean age 36 years; age 18-62 years; M: $\mathrm{F}$ ratio $=1: 1.6)$ with a variety of Crohn's disease phenotypes $(\mathrm{L} 1=8 ; \mathrm{L} 2=6 ; \mathrm{L} 3=12)$ were treated with UST. 9 patients (35\%) had stricturing disease and 5 patients (19\%) penetrating disease. All patients had failed at least one anti-TNF agent. 15 patients $(58 \%)$ had failed two anti-TNF agents, and $11(42 \%)$ had failed an anti-TNF and subsequent vedolizumab therapy. 7 patients $(27 \%)$ received immunomodulatory co-therapy (AZA $=5 ; \mathrm{MTX}=2)$, and $11(42 \%)$ received bridging steroids.

12 week data was available for 20 patients. At 12 weeks, mean HBI significantly improved (5 vs 9; $\mathrm{p}<0.05$ ). There was reduction in mean FC (763 vs 1026 ; ns), but no change in mean CRP (14 vs 11 ; ns). 10 patients (50\%) demonstrated subjective and objective (FC +/-CRP +/- endoscopic) response to therapy. 6 of these patients received bridging steroids, of which all had reduced and 4 had completed their steroid course. Of all treated patients 2 discontinued UST (recurrence of a transitional cell carcinoma; primary nonresponse to therapy requiring surgery), and side effects were reported in 2 patients (Bell's Palsy; lower respiratory tract infection). 
Conclusions UST appears clinically effective and safe in this cohort of treatment-refractory Crohn's disease patients after 12 weeks of therapy. Future work to combine 'real world' data and to assess longer term outcomes will help us to better understand and place the use of UST in the management of Crohn's disease.

\section{PWE-009 THE ACCURACY OF FAECAL CALPROTECTIN MEASUREMENT FROM STOMA EFFLUENT IN PREDICTING CROHN'S DISEASE ACTIVITY}

Sonia Bouri ${ }^{*}$, Jonathan Segal, Siddhartha Oke, Simon Gabe, Ailsa Hart. St Mark's Hospital, London, UK

\subsection{6/gutjnl-2018-BSGAbstracts. 141}

Introduction Faecal Calprotectin (FC) is a marker of neutrophil activity, and is sensitive at detecting gastrointestinal inflammation. FC levels $>150 \mu \mathrm{g} / \mathrm{g}$ are considered to be associated with a higher risk of endoscopically active inflammatory bowel disease. Meta-analyses report that the sensitivity and specificity at predicting active Crohn's disease (CD) using a cut off $\mathrm{FC}>50 \mu \mathrm{g} / \mathrm{g}$ is $83 \%-91 \%$ and $47 \%-53 \%$ respectively; for $\mathrm{FC}>150 \mu \mathrm{g} / \mathrm{g}$, the sensitivity and specificity is $75 \%$ and $71 \%$ respectively. Whilst the use of FC from stoma effluent has been studied in the context of predicting allograft rejection after small bowel transplant, its use in IBD has not been assessed, even though many $\mathrm{CD}$ patients have stomas. The aim of this study is to assess the accuracy of FC from stoma samples.

Methods Consecutive patients with a stoma and CD were identified from a prospectively maintained clinical database. The FC from stoma effluent was categorised as: $\mathrm{FC}<50 \mu \mathrm{g} /$ g, $50-100 \mu \mathrm{g} / \mathrm{g}, 100-150 \mu \mathrm{g} / \mathrm{g}$ and $>150 \mu \mathrm{g} / \mathrm{g}$. This was correlated to endoscopic and/or radiologic findings within 3 months of the FC result. An endoscopy was considered abnormal if the Simple Endoscopic Score for Crohn's Disease (SES-CD) was $\geq 2$ as rated by 2 blinded observers on the basis of the endoscopy report/pictures. An MRI or CT was considered abnormal if any evidence of active inflammation was reported.

Results 47 FC results were analysed from 29 CD patients with a stoma (M:F 12:15). 16/29 patients had intestinal failure. 25 samples were from an ileostomy, 18 from a jejunostomy and 4 from a colostomy. 18 patients had one sample, 5 had 2 samples, 3 had 3 samples and 2 patients had 4 samples assessed. The median time between FC and imaging was 40 days (range 2-95); and between FC and endoscopy was 56 days (range 6-91). 29 patients had a CT or MRI, 9 had an endoscopy and 9 had both. Of the 4 colostomy samples: 3 had FC of $<150 \mu \mathrm{g} / \mathrm{g}$ and none had evidence of active disease; one had a FC of $1130 \mu \mathrm{g} / \mathrm{g}$ and moderate inflammation of a jejunal segment on endoscopy. Of the 43 ileostomy samples: 29 had FC $<150 \mu \mathrm{g} / \mathrm{g}$, of which $3 \mathrm{had}$ active disease (false negatives).

The sensitivity at FC cut off of $50 \mu \mathrm{g} / \mathrm{g}$ and $150 \mu \mathrm{g} / \mathrm{g}$ was $80 \%$ and $73 \%$ respectively. The sensitivity, specificity, positive predictive value (PPV) and negative predictive value (NPV) for three FC cut offs are shown in table 1.
Abstract PWE-009 Table 1 The accuracy of FC at predicting small bowel Crohn's disease activity

\begin{tabular}{lllll}
\hline $\begin{array}{l}\text { Stoma FC cut } \\
\text { off }\end{array}$ & Sensitivity & Specificity & PPV & NPV \\
\hline$<50 \mu \mathrm{g} / \mathrm{g}$ & $80 \%$ & $47 \%$ & $41 \%$ & $83 \%$ \\
$<100 \mu \mathrm{g} / \mathrm{g}$ & $80 \%$ & $78 \%$ & $63 \%$ & $89 \%$ \\
$<150 \mu \mathrm{g} / \mathrm{g}$ & $73 \%$ & $91 \%$ & $79 \%$ & $88 \%$ \\
\hline
\end{tabular}

Conclusion We report that stoma FC has a sensitivity and specificity which is similar to stool FC at all three cut offs. These results suggest that FC is a useful adjunct to clinical assessment and investigations, and a prospective trial in which there is a shorter interval between FC and the diagnostic test is required.

\section{PWE-010 DEFINING INTERLEUKIN-27 EFFECTS ON THE EPITHELIAL BARRIER - A NEW THERAPEUTIC FOR IBD?}

Daniel P Brice*, Susan H Berry, Heather M Wilson, Graeme I Murray, Mairi H McLean. School of Medicine, Medical Sciences and Nutrition, University Of Aberdeen, UK

\subsection{6/gutjnl-2018-BSGAbstracts. 142}

Introduction There is a clinical need for new and safer treatment options for inflammatory bowel disease (IBD). Interleukin-27 (IL-27) is an endogenous immunosuppressive cytokine through inhibition of Th2 and Th17 T cell responses and promotion of IL-10 secreting $\operatorname{Tr} 1 \mathrm{~T}$ regulatory cells. Oral IL-27 clinically attenuates disease in several $\mathrm{T}$ cell and innate cell driven pre-clinical models of IBD. We need to translate this pre-clinical data to human IBD to underpin proof of concept for IL-27 as a new therapeutic for IBD. Our aim was to define IL-27 evoked responses in the human colonic epithelial barrier.

Methods A human colon derived epithelial organoid model was established from fresh colon tissue obtained through the Grampian Tissue Biorepository. Ethical approval was granted by the Biorepository Scientific Access Group. Isolated colonic crypts were grown in a $3 \mathrm{D}$ matrix with conditioned media and growth factors to maturity. RT-qPCR using TaqMan probes characterised organoid permeability gene expression (CLDN2, CLDN4, OCLN, CDH1, TJP1, ECM1) normalised to $G A P D H$ and $B 2 M$ following a 48 hour stimulation with recombinant IL-27 (rIL-27) (0, 50, $100 \mathrm{ng} / \mathrm{ml})$. Data was analysed with the Livak method and one-way ANOVA. The impact of rIL-27 on colonic epithelial growth dynamics was established on Caco-2 and HT-29 cell lines, stimulated with 0, 50 and $100 \mathrm{ng} / \mathrm{ml} \mathrm{rIL-27,} \mathrm{defined} \mathrm{by} \mathrm{(1)} \mathrm{wound} \mathrm{scratch} \mathrm{assays}$ over 72 hours with digital images of wound areas captured at 24 hour intervals and analysed with Image J software, and (2) tritiated thymidine incorporation proliferation assays stimulated for 48 hours.

Results rIL-27 evoked a differential epithelial permeability gene expression in human colonic organoids, with reduced expression of CLDN2 $(\mathrm{p}<0.01)$ and increased expression of CLDN4, OCLN, CDH1 and TJP1 $(\mathrm{p}<0.001)$ in a dose-responsive manner. rIL-27 led to an increased rate in epithelial wound restitution $(\mathrm{p}<0.001)$. In keeping with this, stimulation with rIL-27 